\title{
Effect of dispersion corrections on ab initio predictions of graphite and diamond properties under pressure
}

\author{
Igor Mosyagin,,${ }^{1,2}$ Davide Gambino, ${ }^{1}$ Davide G. Sangiovanni,,${ }^{3,1, *}$ Igor A. Abrikosov,,${ }^{1,2}$ and Nuala M. Caffrey ${ }^{4,1}$ \\ ${ }^{1}$ Department of Physics, Chemistry and Biology (IFM), Linköping University, SE-581 83, Linköping, Sweden \\ ${ }^{2}$ Materials Modeling and Development Laboratory, NUST “MISIS”, 119049 Moscow, Russia \\ ${ }^{3}$ Atomistic Modelling and Simulation, ICAMS, Ruhr-Universität Bochum, D-44801 Bochum, Germany \\ ${ }^{4}$ School of Physics and CRANN, Trinity College, Dublin 2, Ireland
}

(Received 4 September 2018; published 8 November 2018)

\begin{abstract}
There are several approaches to the description of van der Waals (vdW) forces within density functional theory. While they are generally found to improve the structural and energetic properties of those materials dominated by weak dispersion forces, it is not known how they behave when the material is subject to an external pressure. This could be an issue when considering the pressure-induced structural phase transitions, which are currently attracting great attention following the discovery of an ultrahard phase formed by the compression of graphite at room temperature. In order to model this transition, the functional must be capable of simultaneously describing both strong covalent bonds and weak dispersion interactions as an isotropic pressure is applied. Here, we report on the ability of several dispersion-correction functionals to describe the energetic, structural, and elastic properties of graphite and diamond, when subjected to an isotropic pressure. Almost all of the tested vdW corrections provide an improved description of both graphite and diamond compared to the local density approximation. The relative error does not change significantly as pressure is applied, and in some cases even decreases. We therefore conclude that the use of dispersion-corrected exchange-correlation functionals, which have been neglected to date, will improve the accuracy and reliability of theoretical investigations into the pressure-induced phase transition of graphite.
\end{abstract}

DOI: 10.1103/PhysRevB.98.174103

\section{INTRODUCTION}

Diamond and graphite are two of the best known allotropes of carbon. While the former is known for its extreme hardness, making it a highly valuable industrial component in many applications, the latter can be classified as a soft material with uses in lubrication and pencil lead. The differing mechanical behavior can be related to their respective crystalline structures. The carbon atoms in a diamond lattice are $s p^{3}$ hybridized in a tetrahedral geometry. This isotropic bonding configuration gives rise to an exceptional hardness. In contrast, graphite consists of planar sheets of $s p^{2}$ hybridized carbon atoms, with relatively weak dispersion forces holding the layers together. This anisotropic bonding means the layers can glide easily over each other, resulting in a macroscopic softness.

While graphite is the stable phase of carbon under normal conditions, the free-energy difference between it and diamond is only $30 \mathrm{meV}$ [1]. Despite this, it is very difficult to convert graphite into diamond. Temperatures greater than $1700 \mathrm{~K}$ and pressures higher than $12 \mathrm{GPa}$ are required to overcome the free-energy barrier preventing the spontaneous transition. The phase diagram of solid carbon also includes nanotubes, fullerenes, and amorphous $s p^{2}$ and $s p^{3}$ bonded carbon.

Several other phases emerge upon the application of an external pressure, including a metastable, crystalline,

\footnotetext{
*davsan@ifm.liu.se
}

superhard, and transparent phase which is formed by the compression of graphite at room temperature [2-8]. The exact structure of this material is under considerable debate. Structure prediction methods, such as minima hopping, evolutionary algorithms, transition path sampling, and particle-swarm optimization, have suggested several contenders, including body-centered tetragonal $\mathrm{C}_{4}$-carbon [9], orthorhombic $O$ carbon [10], $W$-carbon [10], $Z$-carbon [11], and monoclinic $M$-carbon [12,13], amongst others [14-17].

Those structure prediction methods based on firstprinciples density functional theory (DFT) generally employ standard local or semilocal approximations to the exchangecorrelation energy in order to describe the electronic structure. However, such methods are inherently unable to describe the nonlocal dispersion interactions which dominate the interlayer binding of the starting material, graphite. While the local-density approximation (LDA) gives structural parameters which agree with experiment $[18,19]$, this is a fortuitous agreement that masks an incomplete description of the interlayer van der Waals (vdW) interaction [20]. The generalized gradient approximation (GGA) fails to predict any graphite interlayer characteristics correctly, including its interlayer distance, binding energy, elastic constants, and phonon dispersions $[21,22]$. Thus, neither LDA nor GGA are suitable for an accurate description of graphite under pressure.

Mao et al. showed that the room-temperature (RT) compression of graphite caused approximately half of its $\pi$ bonds to convert to $\sigma$ bonds, with the other half remaining as $\pi$ bonds [6]. Therefore, in order to correctly describe 
pressure-induced transitions of graphite on the atomic scale, one needs to be capable of simultaneously describing both strong covalent bonds $\left(s p^{2}\right.$ and $\left.s p^{3}\right)$ and weak dispersion interactions. Several functionals have been developed to improve the description of dispersion interactions in layered materials, with good results [23-31]. The effect of those functionals developed to describe vdW binding have also been applied to diamond and other ionic or semiconducting solids. In the considered cases, an improved agreement with experimental data has been found [32-38].

However, few first-principles calculations go beyond a determination of the equilibrium properties at zero external pressure. Yu et al. determined the structural properties of diamond and graphite under an applied pressure using the semiempirical correction of Grimme [39]. In this study, we extend this investigation to determine how well more sophisticated vdW-corrected functionals describe both $\mathrm{AB}$-stacked hexagonal graphite and cubic diamond under an applied pressure. We determine their structural and elastic properties as well as how the stacking sequence of the carbon layers in graphite, namely hexagonal $\mathrm{AB}$, rhombohedral $\mathrm{ABC}$, and hexagonal AA, depends on the applied pressure. As the ultimate goal is to perform large-scale, finite-temperature simulations of the phase transition using ab initio molecular dynamics, it is important that a modest computational expense is maintained. For this reason, we restrict our investigation to six different vdW corrected functionals which are of reasonable computational cost, and do not consider more advanced functionals such as the many-body dispersion energy method of Tkatchenko et al. [40,41]. The main details of the employed functionals will be outlined in the following section.

\section{THEORETICAL METHODS}

\section{A. Van der Waals functionals}

We consider four different functionals which account for the dispersion interaction via a pair-wise additive correction to the total energy, namely, the semiempirical methods developed by Grimme (PBE+D3) [42], both with and without Becke-Johnson (BJ) damping (PBE+D3+BJ) [43] and two nonempirical methods developed by Tkatchenko and Scheffler: the standard method (PBE+TS) [44] and with the inclusion of a self-consistent screening (PBE+TS+SCS) [40].

In these functionals, the total energy $E_{\text {total }}$ can be expressed as follows:

$$
E_{\text {total }}=E_{\mathrm{KS}-\mathrm{DFT}}+E_{\mathrm{disp}},
$$

where $E_{\mathrm{KS}-\mathrm{DFT}}$ is the conventional Kohn-Sham DFT energy and $E_{\text {disp }}$ is the energy correction due to the dispersion interaction.

For the two Grimme methods, $E_{\text {disp }}$ takes the form of a damped atom-pair-wise additive correction, such that

$$
E_{\mathrm{disp}}=-\frac{1}{2} \sum_{i} \sum_{j} \frac{C_{6, i j}}{R_{i j}^{6}} f_{\mathrm{damp}}\left(R_{i j}\right),
$$

where $C_{6, i j}$ is the dispersion coefficient for atom pair $i j$ which depends on the local geometry via the coordination number of the atoms and $R_{i j}$ is the distance between them. The damping term $f_{\text {damp }}$ is to ensure that the potentials do not diverge at short distances. $\mathrm{PBE}+\mathrm{D} 3+\mathrm{BJ}$ applies a correction to the damping function of $\mathrm{PBE}+\mathrm{D} 3$ to ensure the dispersion energy approaches a constant value at small $R$.

The two functionals developed by Tkatchenko and Scheffler, on the other hand, include the dispersion interaction as pair-wise potentials proportional to the rescaled polarizabilities of the free atoms. These are obtained from selfinteraction corrected time-dependent DFT calculations [44]. The updated PBE+TS+SCS functional further accounts for electrodynamic response effects by solving the self-consistent screening (SCS) equation of electrodynamics for a dipole field of quantum harmonic oscillators [40].

We also consider two vdW functionals (optB86b [45] and rev-vdW-DF2 [46]) which, rather than account for vdW interactions via an additive correction to the total energy, include a nonlocal correlation energy that encompasses the long-range interactions. Here, the exchange correlation energy is defined as

$$
E_{\mathrm{xc}}=E_{\mathrm{x}}^{\mathrm{GGA}}+E_{\mathrm{c}}^{\mathrm{LDA}}+E_{\mathrm{c}}^{\mathrm{nl}},
$$

where $E_{\mathrm{x}}^{\mathrm{GGA}}$ is the GGA exchange energy and $E_{\mathrm{c}}^{\mathrm{LDA}}$ is the local correlation within LDA. The remaining term, $E_{\mathrm{c}}^{\mathrm{nl}}$, is a nonlocal correlation energy based on a model response function of interacting electron densities [47]. In the original formulation of Dion et al., the exchange energy was that of Perdew-Burke-Ernzerhof (revPBE) [48]. However, it was found to consistently overestimate equilibrium separations for the S22 benchmark set of molecular duplexes [49]. The optB86b method of Klimeš et al. replaces revPBE with a version of the B86b exchange functional [50] which was optimized by fitting to the S22 data set. A substantial improvement in accuracy was achieved $[32,45]$. We also employ the rev-vdW-DF2 functional developed by Hamada [46] which uses a revised B86b exchange functional together with the nonlocal correlation functional of vdW-DF2 [51]. An improved performance for a wide range of materials, including graphite and other layered materials, was found.

However, it is not clear how transferable this category of functionals are to systems beyond those for which they were designed. For example, they were shown to achieve good agreement for some systems including graphite and diamond [32,35], but to behave poorly when applied to the intralayer thickness of a variety of layered materials [26].

\section{B. Computational details}

Kohn-Sham DFT calculations were carried out using the projector augmented wave method [52] as implemented in VASP-5.3 [53-57]. Brillouin-zone integration was carried out using the Monkhorst-Pack method [58]. Convergence tests found that a cutoff energy of $1000 \mathrm{eV}$ and a $23 \times 23 \times 11$ $k$-point grid are required to describe $\mathrm{AB}$-stacked graphite. The diamond structure was converged using a $9 \times 9 \times 9 k$ point mesh. Structural optimizations were performed using the conjugate-gradient algorithm until the forces smaller than $1 \mathrm{meV} / \AA$ were achieved.

To obtain bulk properties, the total energies were calculated for a set of volumes corresponding to an increased isotropic pressure ranging between 0 and $30 \mathrm{GPa}$. Energy-volume data sets were then fitted using the Murnaghan equation of state 
TABLE I. Bulk properties of graphite and diamond at 0 GPa. MARE is defined in Eq. (5) using three descriptors for graphite $\left(a_{0}, c_{0}\right.$, and $\left.B_{0}\right)$ and two descriptors for diamond $\left(a_{0}\right.$ and $\left.B_{0}\right)$. For the case of graphite, the data are compared to the experimental data taken at $4.2 \mathrm{~K}$. For the case of diamond, the theoretical data are compared to experimental data taken from Ref. [65], which has already been corrected for thermal expansion and zero-point motion.

\begin{tabular}{|c|c|c|c|c|c|c|c|c|c|}
\hline \multicolumn{6}{|c|}{ Graphite } & \multicolumn{4}{|c|}{ Diamond } \\
\hline Functional & $a_{0}(\AA)$ & $c_{0}(\AA)$ & $c_{0} / a_{0}$ & $B_{0}(\mathrm{GPa})$ & MARE & Functional & $a_{0}(\AA)$ & $B_{0}(\mathrm{GPa})$ & MARE \\
\hline LDA & 2.447 & 6.652 & 2.719 & 25.5 & 9.2 & LDA & 3.535 & 467.0 & 2.8 \\
\hline $\mathrm{PBE}+\mathrm{D} 3$ & 2.465 & 6.965 & 2.825 & 23.2 & 12.7 & $\mathrm{PBE}+\mathrm{D} 3$ & 3.564 & 442.4 & 0.4 \\
\hline $\mathrm{PBE}+\mathrm{D} 3+\mathrm{BJ}$ & 2.465 & 6.745 & 2.736 & 30.4 & 4.7 & $\mathrm{PBE}+\mathrm{D} 3+\mathrm{BJ}$ & 3.557 & 445.3 & 0.5 \\
\hline $\mathrm{PBE}+\mathrm{TS}$ & 2.459 & 6.690 & 2.721 & 53.5 & 17.9 & $\mathrm{PBE}+\mathrm{TS}$ & 3.552 & 443.8 & 0.2 \\
\hline $\mathrm{PBE}+\mathrm{TS}+\mathrm{SCS}$ & 2.462 & 6.707 & 2.725 & 36.1 & 1.4 & $\mathrm{PBE}+\mathrm{TS}+\mathrm{SCS}$ & 3.563 & 439.8 & 0.6 \\
\hline rev-vdW-DF2 & 2.464 & 6.608 & 2.682 & 44.7 & 9.7 & rev-vdW-DF2 & 3.567 & 421.1 & 2.8 \\
\hline optB86b & 2.466 & 6.620 & 2.685 & 33.8 & 1.4 & optB86b & 3.569 & 424.2 & 2.5 \\
\hline Expt. [63] (4.2 K) & 2.459 & 6.672 & 2.713 & 34.9 & & Expt. [65] & 3.543 & & \\
\hline Expt. [64] (RT) & 2.462 & 6.707 & 2.724 & $34-42$ & & Expt. [66] (RT) & 3.567 & 443.0 & \\
\hline
\end{tabular}

(EOS):

$$
E(V)=E\left(V_{0}\right)+\frac{B_{0} V}{B_{0}^{\prime}}\left(\frac{\left(V_{0} / V\right)^{B_{0}^{\prime}}}{B_{0}^{\prime}-1}+1\right)-\frac{B_{0} V_{0}}{B_{0}^{\prime}-1},
$$

which yields bulk moduli $B_{0}$, equilibrium volumes $V_{0}$, and bulk modulus derivatives $B_{0}^{\prime}$. Additionally, one obtains $E_{0}=$ $E\left(V_{0}\right)$ as an equilibrium energy. Graphite has two independent structural parameters: the in-plane lattice constant $a_{0}$ and the interplanar distance $c_{0}$. Cubic diamond can be described with only one independent lattice parameter, $a_{0}$.

We also determine the elastic constants for both diamond and graphite at $0 \mathrm{GPa}$, using the built-in VASP routine based the infinitesimal deformation method $[59,60]$. The zero-point energy was calculated as ZPE $=\frac{\hbar}{2} \int \omega g(\omega) d \omega$, where $g(\omega)$ is the vibrational density of states and $\omega$ is the frequency. This was determined within the harmonic approximation as implemented in the PHONOPY package [61].

\section{RESULTS}

As we have mentioned, the structural properties of both graphite [23-31,62] and to a lesser extent, diamond [32-37], have been investigated previously at ambient pressure using a variety of vdW-corrected functionals. Here, we repeat these calculations with the aim of ensuring consistency across the range of functionals used. We then extend our study to determine how well these functionals describe both graphite and diamond as an isotropic external pressure is applied.

\section{A. Zero pressure}

The ground-state structural properties of both graphite and diamond at $0 \mathrm{GPa}$ are presented in Table I for LDA and the six considered dispersion-corrected functionals and compared to available experimental data. In order to determine the quality of the performance of different functionals in comparison with experiment over a variety of different descriptors such as lattice constants and bulk moduli, we define the mean absolute relative error (MARE) achieved by a functional as

$$
\text { MARE }=\frac{100}{n} \sum_{t=1}^{n}\left|\frac{\xi_{\text {expt }}-\xi_{\text {theo }}}{\xi_{\text {expt }}}\right|,
$$

where $n$ is the number of descriptors being considered, $\xi_{\text {expt }}$ is the experimental value of the descriptor, and $\xi_{\text {theo }}$ is its theoretical value.

We find that all of the vdW-inclusive functionals give an improvement over LDA when considering the in-plane lattice constant of graphite, $a_{0}$ : while LDA underestimates it by $0.5 \%$ compared to the low-temperature experimental value, each of the vdW functionals give errors of less than $0.3 \%$.

Mounet and Marzari calculated the effects of zero-point motion on the structural properties of graphite, and found that the $a_{0}$ lattice constant increases by $0.0074 \AA$ [22]. In this case, the contribution was determined using the PBE functional and the experimental $c / a$ ratio of graphite. However, we find that the zero-point energy varies by less than $1.3 \%$ when calculated with LDA, TS+SCS, and the MBD (at $0.17 \mathrm{meV} / \mathrm{C}$ ). Similarly, Zhang et al. found that the effect of the specific functional on the zero-point energy was negligible when considering a database of bulk solids [38]. We find that the vdW-corrected functionals actually increase in error compared to LDA when the contribution of zero-point motion is included. The error associated with the in-plane lattice constant as determined by the LDA functional is $0.2 \%$ when corrected for zero-point motion, while all other functionals overestimate the $a_{0}$ by up to $0.6 \%$. Nonetheless, these errors are relatively small and so we can conclude that all of the considered vdW functionals describe $a_{0}$ well.

Some vdW-inclusive functionals perform worse than LDA in describing the out-of-plane lattice parameter of graphite, $c_{0}$. In particular, the PBE $+\mathrm{D} 3$ functional overestimates $c_{0}$ by $4.4 \%$ compared to an error of $0.3 \%$ in LDA. The best performing functionals are those of Tkatchenko and Scheffler, with errors of less than $0.3 \%(0.5 \%)$ for PBE+TS (PBE+TS+SCS). The $\mathrm{PBE}+\mathrm{D} 3+\mathrm{BJ}$ functional gives an error of $1.1 \%$, while the two nonlocal functionals (rev-vdW-DF2 and optB86b) both underestimate $c_{0}$ by $1 \%$ and $0.8 \%$, respectively. If zero-point motion is included to the extent determined by Mounet and Marzari (3\%), those functionals which underestimated the $c_{0}$ lattice constant all improve with respect to experiment (i.e., LDA and the two nonlocal functionals) while those functionals which overestimate the lattice constant all increase in error by $0.3 \%$. As a result, LDA is in fact found to fortuitously reproduce the experimental value exactly. 
TABLE II. Elastic constants of graphite and diamond at $0 \mathrm{GPa}$. All values are in GPa. MAPE is defined in Eq. (6).

\begin{tabular}{|c|c|c|c|c|c|c|c|c|c|c|c|c|}
\hline Functional & $C_{11}$ & $C_{12}$ & $C_{13}$ & $C_{33}$ & $C_{44}$ & $C_{66}$ & MAPE & Functional & $C_{11}$ & $C_{12}$ & $C_{44}$ & MAPE \\
\hline $\mathrm{PBE}+\mathrm{D} 3$ & 1040 & 198 & -2.4 & 26 & 3.0 & 421 & 2.6 & $\mathrm{PBE}+\mathrm{D} 3$ & 1064 & 132 & 579 & 1.6 \\
\hline $\mathrm{PBE}+\mathrm{D} 3+\mathrm{BJ}$ & 1095 & 218 & -2.6 & 34 & 4.2 & 439 & 1.6 & $\mathrm{PBE}+\mathrm{D} 3+\mathrm{BJ}$ & 1072 & 132 & 588 & 2.6 \\
\hline PBE+TS & 1071 & 193 & -7.2 & 66 & 5.5 & 439 & 2.5 & $\mathrm{PBE}+\mathrm{TS}$ & 1075 & 128 & 588 & 2.1 \\
\hline optB86b & 1087 & 166 & -3.4 & 42 & 5.5 & 461 & 1.0 & optB86b & 1052 & 129 & 564 & 4.7 \\
\hline Expt. [67] & $1109 \pm 16$ & $139 \pm 36$ & $0 \pm 3$ & $38.7 \pm 7$ & $5 \pm 3$ & $485 \pm 10$ & & Expt. [68] & $1081 \pm 5$ & $125 \pm 5$ & $579 \pm 2$ & \\
\hline
\end{tabular}

The agreement between theory and experiment is not as close when considering the bulk modulus. LDA fails to reproduce the experimental value, underestimating it by approximately $27 \%$. PBE+D3 gives a similarly large error of $33 \%$. The inclusion of $\mathrm{BJ}$ damping reduces this error to $13 \%$. The PBE+TS functional, on the other hand, describes graphite as a much harder material than experimentally found, with a bulk modulus of $53.5 \mathrm{GPa}$, an overestimation of over $50 \%$. This is in agreement with previous work [24]. A dramatic improvement occurs with the inclusion of the SCS correction, reducing the error to only $3.4 \%$. A similar error is achieved with the optB86b functional. However, the rev-vdW-DF2 functional also performs poorly, with an error of $28 \%$. If we correct for zero-point motion, taking an increase in $B_{0}$ of approximately $1 \mathrm{GPa}$ [22], the best performing functional is that of PBE+TS+SCS with an error of only $0.6 \%$.

In fact, the best performance across all descriptors is given by $\mathrm{PBE}+\mathrm{TS}+\mathrm{SCS}$ and optB86b, with a MARE value of $1.4 \%$, followed by the PBE+D3+BJ $(4.7 \%)$ functional. These three functionals remain the best performers when zero-point corrections are taken into account.

Turning now to diamond, all functionals perform well in the description of the bulk lattice constant. The smallest error is again given by LDA (an underestimation of $0.2 \%$ ). The vdW-corrected functionals all overestimate the lattice constant, but the error is never larger than $0.7 \%$ (that given by the two nonlocal functionals.) In this case, the lattice constant was compared to an experimental value of $3.543 \AA$ which has been corrected for both thermal expansion at room temperature and zero-point motion [65].

The four $\mathrm{C}_{6}$-corrected functionals are found to reduce the error associated with the bulk modulus of diamond compared to LDA, from $5.4 \%$ to less than $0.7 \%$. The bulk modulus as determined with rev-vdW-DF2 and optB86b is underestimated by approximately $4 \%$. Taking zero-point motion into account, which serves to reduce the bulk modulus by approximately $10 \mathrm{GPa}$ at $0 \mathrm{~K}$ [22], the error in all of the vdW-corrected functionals increases by $2.3 \%$.

The results presents here for ambient pressure agree with those presented in the literature [28-30,32,35]. Given that a good description of graphite and diamond is required simultaneously, we find that the PBE+TS+SCS is the best performing functional (with a MARE value of $1.0 \%$ ), while both $\mathrm{PBE}+\mathrm{D} 3+\mathrm{BJ}$ and optB86b also perform reasonably well (with MARE values between $1.9 \%$ and 2.6\%). The large overestimation of the $c$ lattice constant of graphite using $\mathrm{PBE}+\mathrm{D} 3$ and the large overestimation of the graphite bulk modulus using PBE+TS make these two functionals less accurate, with MARE values of just under 10\%, and indeed, perform worse than LDA with a MARE of $6 \%$. The inclusion of zero-point motion increases the error slightly, but the designation of $\mathrm{PBE}+\mathrm{TS}+\mathrm{SCS}, \mathrm{PBE}+\mathrm{D} 3+\mathrm{BJ}$, and optB $86 \mathrm{~b}$ as the best performing functionals is not changed.

\section{B. Elastic properties}

The elastic constants of graphite and diamond at $0 \mathrm{GPa}$ as calculated with the different dispersion corrected functionals are presented in Table II and compared to experiment. Given that the values of some of the elastic constants are small, we define a different quality criterion to estimate the performance of the considered functionals. The mean absolute percentage error (MAPE) is defined as

$$
\text { MAPE }=\frac{1}{n} \sum_{t=1}^{n}\left|\frac{\xi_{\text {expt }}-\xi_{\text {theo }}}{\varepsilon_{\text {expt }}}\right|,
$$

where $n$ is the number of elastic constants considered, $\xi_{\text {expt }}$ is the experimental value, $\xi_{\text {theo }}$ is the theoretical value, and $\varepsilon_{\text {expt }}$ is the reported experimental error, given by the corresponding error bars. Using this definition, theoretical values lying within the experimental error range give a MAPE between 0 and 1.

We find that the elastic constants of graphite are best described by the two nonlocal functionals, rev-vdW-DF2 and optB86b, which both give results within the experimental range. The largest error is given by the $\mathrm{PBE}+\mathrm{D} 3$ functional $(2.6 \%)$, followed by the two Tkatchenko-Scheffler functionals. $\mathrm{PBE}+\mathrm{D} 3+\mathrm{BJ}$ and LDA give similar errors of approximately $1.5 \%$.

For the case of diamond, LDA unsurprisingly yields substantial disagreement with experiment, with an absolute error of $7.4 \%$. The best results are obtained with the $C_{6}$ corrected functionals, with errors of between $1.6 \%$ and $2.6 \%$, while the nonlocal functionals perform somewhat worse with errors of almost 5\%. However, these errors would represent the typical accuracy of elastic constants calculated with DFT.

Summarizing this section, we see that the best functionals for describing simultaneously the elastic constants of both graphite and diamond are the two Grimme functionals with 

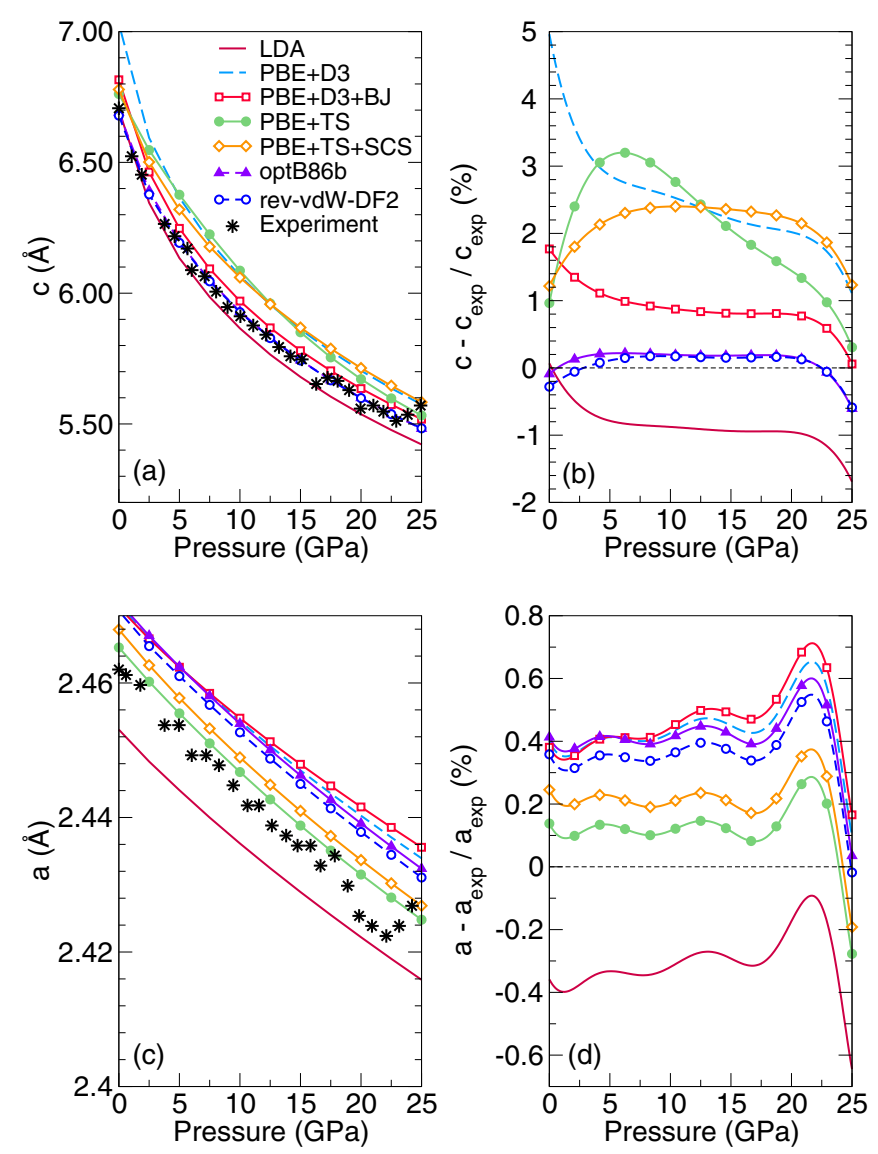

FIG. 1. (a) Out-of-plane $c$ lattice parameter of graphite as calculated with different vdW-corrected functionals as a function of pressure and compared to experimental data (black stars). (b) The relative deviation of the calculated $c$ values from the experimental data. (c) In-plane $a$ lattice parameter of graphite compared to experiment. The experimental data are taken from Ref. [64]. (d) The relative deviation of the calculated $a$ values from the experimental data. Note that in all cases the theoretical data have been corrected for thermal expansion and zero-point motion, as described in the text.

a combined MARE value of just over $2 \%$. However, the other vdW-corrected functionals also perform well, with an error of no more than $3 \%$, which is an improvement over the LDA error. These results are in good agreement with other theoretical works $[22,69]$.

\section{Pressure dependence}

We now consider how these functionals behave when an external pressure is applied to both graphite and diamond. Figures 1(a) and 1(b) show how the $c$ lattice constant of graphite behaves as the external pressure is increased from 0 to $25 \mathrm{GPa}$ for each of the considered functionals. This is then compared to the experimental data obtained by Wang et al. at room temperature [8]. In order to ensure a fair comparison, the theoretical data have been shifted to take zero-point motion and thermal expansion into account. The out-of-plane linear thermal expansion coefficient is $27 \times 10^{-6} \mathrm{~K}^{-1}$ at room temperature [70]. This corresponds to an increase in the $c$ lattice constant of $0.05 \AA$ as the temperature is raised to room temperature. The expansion of the $c$ lattice due to zero point motion is $0.02 \AA$ [22]. As a result, the $0 \mathrm{~K}$ results are shifted by $0.07 \AA$ and the results compared to the experimental data. Likewise, the theoretical data for the $a$ lattice constant have been shifted by $0.0064 \AA$, taking the linear expansion coefficient of $-1.5 \times 10^{-6} \mathrm{~K}^{-1}$ at room temperature [70], as well as a zero-point expansion of $0.02 \AA$ [22].

Note that above 19.2 GPa the experimental data showed evidence of a phase transition which was completed by $37 \mathrm{GPa}$. Due to the free-energy barrier, our theoretical calculations cannot reproduce this phase transition. Thus, beyond approximately $19 \mathrm{GPa}$ we do not expect good agreement between the computational and experimental lattice constants. In this pressure range, experiment shows a decrease in both the $a$ and $c$ lattice parameters compared to the calculated values.

All of the functionals show the $c$ lattice parameter of graphite to reduce by approximately $1.3 \AA$ when the pressure is increased from 0 to $25 \mathrm{GPa}$. This corresponds to a reduction in the interlayer distance of $0.65 \AA$.

As stated earlier, when corrected for zero-point motion, LDA predicts the $c$ lattice constant exactly at $0 \mathrm{GPa}$. However, the error increases with the application of pressure to just under $1 \%$ across a wide pressure window [Fig. 1(b)]. The best performance is achieved by the two nonlocal functionals which behave very similarly and give an error of no more than $0.2 \%$ across the whole pressure range. While $\mathrm{PBE}+\mathrm{D} 3+\mathrm{BJ}$ gives an error of $1.7 \%$ at $0 \mathrm{GPa}$, this decreases to under $1 \%$ at higher pressures. The error of the two TS functionals initially increases with the application of pressure from about $1 \%$ to a maximum of more than $3.1 \%$ (2.3\%) for PBE+TS $(\mathrm{PBE}+\mathrm{TS}+\mathrm{SCS})$ at $6 \mathrm{GPa}$ before decreasing. The PBE+D3 functional, while significantly overestimating the $c$ lattice constant at $0 \mathrm{GPa}$, performs better at higher pressures, reducing in error to about $2.5 \%$.

One thing to note is that the thermal expansion and zeropoint motion corrections have been applied uniformly across all pressures. However, it is possible that the effects of thermal expansion become less significant as the pressure is increased. Given the small errors involved here, it is likely that this would serve to reduce the error given by the vdW functionals at higher pressure.

All of the considered functionals reproduce the experimental in-plane lattice constant of graphite, with errors of no more than $0.5 \%$ for all functionals between 0 and $19 \mathrm{GPa}$. This can be seen in Figs. 1(c) and 1(d). In this case, the two TS functionals, which overestimate the experimental lattice constant by about $0.2 \%$ at all investigated pressures, perform slightly better than other functionals.

Next, we determine how the lattice constant of diamond behaves as a function of applied pressure. This is shown in Fig. 2 and compared to available experimental data [64], which was recorded at room temperature. Therefore, the theoretically determined lattice constant was increased by $0.024 \AA$, including a zero-point expansion of $0.013 \AA$ [22].

The lattice constant of diamond is found to decrease linearly with the applied pressure. The errors achieved by each functional at ambient pressure remain approximately constant across the whole pressure window considered. LDA performs quite well, underestimating $a$ by $0.24 \%$, which decreases slightly with the applied pressure. The PBE+TS 

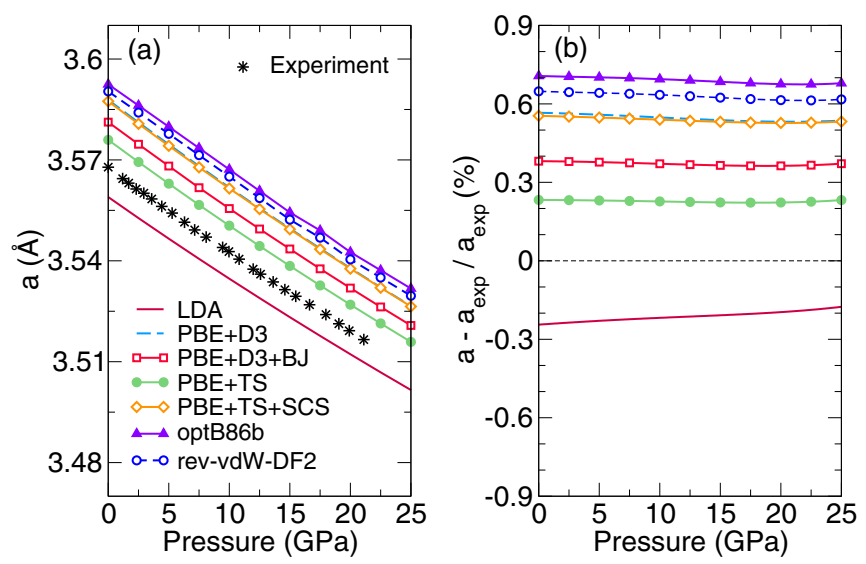

FIG. 2. (a) Lattice constant of diamond calculated with different $\mathrm{vdW}$-corrected functionals as a function of pressure and compared to experimental data taken from Ref. [66]. (b) The corresponding error of the vdW functionals compared to the experimental data. Note that the theoretical data have been corrected for thermal expansion and zero-point motion, as described in the text.

functional also performs well, overestimating the lattice constant by $0.23 \%$. The SCS correction to this functional increases the error to about $0.5 \%$, a comparable error to PBE+D3. The worst performance is achieved by the two nonlocal functionals with errors of approximately $0.7 \%$. In fact, none of the functionals considered in this study give an error of greater than $1 \%$ for the lattice constant of diamond.

We can therefore conclude that the application of an external isotropic pressure does not adversely affect the error achieved by the vdW functionals at ambient pressure.

\section{Interlayer binding}

In graphite, the dispersion interactions affect not only the interlayer distance and binding energies but also the layer stacking sequence. The ground-state structure of graphite is generally accepted to be hexagonal with AB-type stacking and space group $P 6_{3} / m m c$. However, the energy differences between this state and other graphite structures containing different stacking sequences are, in some cases, very small. Rhombohedral graphite, with a stacking sequence of $A B C$, is often experimentally reported as planar defects or nanograins embedded in hexagonal graphite [71,72], and is relatively abundant in natural samples. Furthermore, as different stacking sequences are believed to play an important role as transition states during pressure-induced phase transformations [73], the relative energetics of these graphite polymorphs have attracted increased interest. As such, it is important to determine how well the various dispersion-corrected functionals behave in calculating this quantity.

The interlayer binding energies of graphite in the $A B$, $\mathrm{AA}$, and $\mathrm{ABC}$ stackings are reported in Table III for each of the vdW functionals. For completeness, we now include the PBE+MBD functional, which includes nonlocal many-body dispersion (MBD) [33,41]. This functional has previously been found to give a very accurate description of graphite [29]. The interlayer binding energy of the stacking $E_{b}^{\text {stacking }}$
TABLE III. Graphite interlayer binding energy, $E_{b}$, as calculated with different vdW-corrected functionals for three stacking arrangements of the graphene layers, compared with the value determined by the random phase approximation and some reported experimental data. $\Delta E$ is the energy of a particular stacking with respect to the $\mathrm{AB}$-stacking configuration, defined in Eq. (8). All values are given in meV per atom.

\begin{tabular}{|c|c|c|c|c|c|}
\hline \multirow[b]{2}{*}{ Functional } & \multirow{2}{*}{$\frac{\mathrm{AB}}{E_{b}}$} & \multicolumn{2}{|c|}{$\mathrm{AA}$} & \multicolumn{2}{|c|}{$\mathrm{ABC}$} \\
\hline & & $\overline{E_{b}}$ & $\Delta E$ & $E_{b}$ & $\Delta E$ \\
\hline LDA & 23 & 14 & -9 & 23 & $<1$ \\
\hline $\mathrm{PBE}+\mathrm{D} 3$ & 48 & 42 & -6 & 48 & $<1$ \\
\hline $\mathrm{PBE}+\mathrm{D} 3+\mathrm{BJ}$ & 53 & 44 & -9 & 53 & $<1$ \\
\hline $\mathrm{PBE}+\mathrm{TS}$ & 83 & 69 & -13 & 83 & $<1$ \\
\hline $\mathrm{PBE}+\mathrm{TS}+\mathrm{SCS}$ & 55 & 45 & -10 & 55 & $<1$ \\
\hline rev-vdW-DF2 & 59 & 47 & -11 & 58 & $<1$ \\
\hline optB86b & 70 & 58 & -11 & 70 & $<1$ \\
\hline $\mathrm{PBE}+\mathrm{MBD}$ & 49 & 41 & -9 & 49 & $<1$ \\
\hline RPA (Ref. [74]) & 48 & & -10 & & \\
\hline Expt. (Ref. [76]) & $52 \pm 5$ & & & & \\
\hline Expt. (Ref. [77]) & $31 \pm 2$ & & & & \\
\hline
\end{tabular}

is calculated as

$$
E_{b}^{\text {stacking }}=-\frac{E_{\text {tot }}^{\text {stacking }}-N E_{\text {graphene }}}{n N},
$$

where $E_{\text {tot }}^{\text {stacking }}$ is the total energy of graphite in a particular stacking sequence, $E_{\text {graphene }}$ is the total energy of a isolated graphene sheet, $N$ is the number of layers in the unit cell of each stacking (e.g., $N=2$ for $\mathrm{AB}, N=3$ for $\mathrm{ABC}$ ), and $n$ is the number of atoms in each layer. In addition, we report the energy difference between the chosen stacking and the ground-state $\mathrm{AB}$ structure $\Delta E$. This is defined as

$$
\Delta E=E_{b}^{\text {stacking }}-E_{b}^{\mathrm{ABstacking}} .
$$

The values calculated in this work are compared with available experimental data and with the random phase approximation (RPA) values, taken from Ref. [74]. Compared to the RPA values, the PBE+MBD functional method performs best with an MARE value of $6.0 \%$, while the PBE+TS+SCS and $\mathrm{PBE}+\mathrm{D} 3+\mathrm{BJ}$ functionals lead to a slight increase of the error to $7.3 \%$ and $10.2 \%$, respectively. The PBE+TS functional without self-consistent screening performs the worst with an error of over 50\%. These results are in good agreement with the reported structural and bulk properties since larger binding energies are associated with larger bulk moduli and smaller $c / a$ ratios.

We find that the energy difference between the $\mathrm{ABC}$ and the $\mathrm{AB}$ stackings are approximately $0.1 \mathrm{meV}$. While such small values are in agreement with experiment, which reports stacking fault energies of $0.09 \mathrm{meV} /$ atom [75], the exact value is below the accuracy of our DFT methods. All of the considered functionals predict that $\mathrm{AB}$-stacked graphite has a lower energy than $\mathrm{ABC}$-stacked graphite, apart from $\mathrm{PBE}+\mathrm{TS}+\mathrm{SCS}$ which predicts that $\mathrm{ABC}$ is the ground-state structure. Given that the two stackings are so close in energy, it is common that DFT methods fail to predict comprehensively the correct ground state. Other factors, not considered here, 


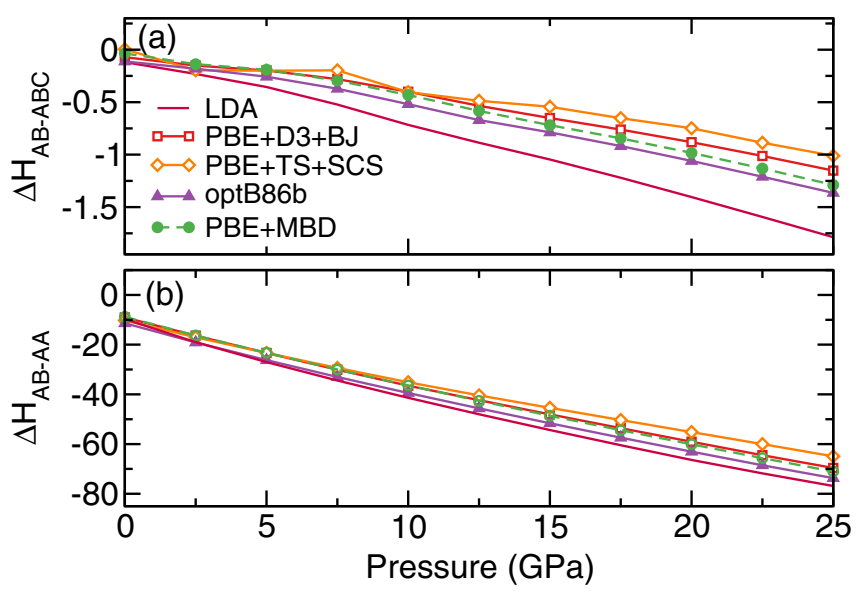

FIG. 3. Enthalpy difference between (a) AB- and ABC-stacked graphite and (b) $\mathrm{AB}$ and $\mathrm{AA}$ graphite as a function of pressure. The enthalpy difference is given in units of $\mathrm{meV}$ per atom.

may also have to be taken into account, including the role of phonons.

It is possible that the stacking sequence of graphite could be influenced by an applied pressure, which in turn could be part of the phase transition pathway. To address this issue, we determined the difference in enthalpy between $\mathrm{AB}$ - and both ABC- and AA-stacked graphite as a function of applied pressure with the three functionals that perform best in describing graphite, namely, PBE+D3+BJ, PBS+TS+SCS, and optB86b, as well as PBE+MBD and LDA. The results are shown in Fig. 3. We find that while the application of pressure stabilizes AB-stacked graphite with respect to AA-stacked graphite, at a rate of approximately $-2.2 \mathrm{meV} / \mathrm{GPa}$, the enthalpy difference between $\mathrm{AB}$ and $\mathrm{ABC}$ graphite remains minuscule, with differences of no more than $1.5 \mathrm{meV}$ over the entire pressure range considered. All four functionals behave similarly, with the greatest (least) stabilization achieved by the LDA (PBE+TS+SCS) functional.

\section{CONCLUSION}

We investigated the ability of six different dispersioncorrected exchange correlation functionals to describe the structural and energetic properties of graphite and diamond as a function of an applied pressure. We included four functionals which include vdW interactions as an additive correction together with two functionals which include a nonlocal correlation term.

When considering the lattice constants, bulk moduli and elastic constants of both graphite and diamond, as well as the interlayer binding energy of graphite, we find that the best performing functional across all descriptors is $\mathrm{PBE}+\mathrm{TS}+\mathrm{SCS}$ with a combined error of just $2.2 \%$. This is followed by the semiempirical $\mathrm{PBE}+\mathrm{D} 3+\mathrm{BJ}$ functional with an error of $4.3 \%$. The two nonlocal functionals give a combined error of between $6 \%$ and $7 \%$. Primarily due to the significant overestimation of the $c$ lattice constant of graphite, the combined error of $\mathrm{PBE}+\mathrm{D} 3$ is high, at $8.6 \%$. The overestimation of both the graphite bulk modulus and interlayer binding energy means that the PBE+TS functional performs overall worse that LDA, with a combined mean absolute error of $13.7 \%$ compared to $9.2 \%$.

This is the case at $P=0 \mathrm{GPa}$, and the relative error to experiment does not change significantly upon the application of an isotropic pressure when considering the structural parameters. This is particularly the case for diamond, and in fact the error decreases slightly when compressing graphite.

Finally, we find that an isotropic pressure stabilizes ABstacked graphite relative to AA-stacked graphite at a rate of $-2.2 \mathrm{meV} / \mathrm{GPa}$ over the pressure range considered. In contrast, the relative energy of $\mathrm{AB}$ - and $\mathrm{ABC}$-stacked graphite is in the range of $1.5 \mathrm{meV}$ over the entire pressure range considered, regardless of the dispersion correction used.

\section{ACKNOWLEDGMENTS}

We are grateful for the support provided by the Swedish Research Council (VR) Grants No. 2015-04391, No. 20144750, and No. 637-2013-7296 and the Swedish Government Strategic Research Area in Materials Science on Functional Materials at Linköping University (Faculty Grant No. SFOMat-LiU No. 2009 00971). Theoretical simulations of structural properties were supported by the Russian Science Foundation (Project No. 18-12-00492). D.G.S. gratefully acknowledges financial support from the Olle Engkvist Foundation, and NMC was supported by a Science Foundation Ireland Starting Investigator Research Grant (No. 15/SIRG/3314). Calculations were performed utilizing supercomputer resources supplied by the Swedish National Infrastructure for Computing (SNIC) at NSC center, and at the supercomputer cluster at NUST "MISIS."
[1] P. Gustafson, An evaluation of the thermodynamic properties and the $P, T$ phase diagram of carbon, Carbon 24, 169 (1986).

[2] G. A. Samara and H. G. Drickamer, Effect of pressure on the resistance of pyrolytic graphite, J. Chem. Phys. 37, 471 (1962).

[3] R. B. Aust and H. G. Drickamer, Carbon: A new crystalline phase, Science 140, 817 (1963).

[4] X. Li and H.-K. Mao, Solid carbon at high pressure: Electrical resistivity and phase transition, Phys. Chem. Miner. 21, 1 (1994).
[5] J. M. Montgomery, B. Kiefer, and K. K. M. Lee, Determining the high-pressure phase transition in highly-ordered pyrolitic graphite with time-dependent electrical resistance measurements, J. Appl. Phys. 110, 043725 (2011).

[6] W. L. Mao, Bonding changes in compressed superhard graphite, Science 302, 425 (2003).

[7] Y. Wang and K. K. M. Lee, From soft to superhard: Fifty years of experiments on cold-compressed graphite, J. Superhard Mater. 34, 360 (2012). 
[8] Y. Wang, J. E. Panzik, B. Kiefer, and K. K. M. Lee, Crystal structure of graphite under room-temperature compression and decompression, Sci. Rep. 2, 520 (2012).

[9] K. Umemoto, R. M. Wentzcovitch, S. Saito, and T. Miyake, Body-Centered Tetragonal $C_{4}$ : A Viable $s p^{3}$ Carbon Allotrope, Phys. Rev. Lett. 104, 125504 (2010).

[10] J.-T. Wang, C. Chen, and Y. Kawazoe, Low-temperature Phase Transformation from Graphite to $s p^{3}$ Orthorhombic Carbon, Phys. Rev. Lett. 106, 075501 (2011).

[11] Z. Zhao, B. Xu, X.-F. Zhou, L.-M. Wang, B. Wen, J. He, Z. Liu, H.-T. Wang, and Y. Tian, Novel Superhard Carbon: C-Centered Orthorhombic $\mathrm{C}_{8}$, Phys. Rev. Lett. 107, 215502 (2011).

[12] Q. Li, Y. Ma, A. R. Oganov, H. Wang, H. Wang, Y. Xu, T. Cui, H.-K. Mao, and G. Zou, Superhard Monoclinic Polymorph of Carbon, Phys. Rev. Lett. 102, 175506 (2009).

[13] S. E. Boulfelfel, A. R. Oganov, and S. Leoni, Understanding the nature of "superhard graphite", Sci. Rep. 2, 471 (2012).

[14] M. Amsler, J. A. Flores-Livas, L. Lehtovaara, F. Balima, S. A. Ghasemi, D. Machon, S. Pailhes, A. Willand, D. Caliste, S. Botti et al., Crystal Structure of Cold Compressed Graphite, Phys. Rev. Lett. 108, 065501 (2012).

[15] M. Alvarez-Murga, P. Bleuet, G. Garbarino, A. Salamat, M. Mezouar, and J.-L. Hodeau, "Compressed Graphite" Formed During $\mathrm{C}_{60}$ to Diamond Transformation as Revealed by Scattering Computed Tomography, Phys. Rev. Lett. 109, 025502 (2012).

[16] Ch. He, L. Z. Sun, and J. Zhong, Prediction of superhard carbon allotropes from the segment combination method, J. Superhard Mater. 34, 386 (2012).

[17] Y. Bai, X. Zhao, T. Li, Z. Lv, S. Lv, H. Han, Y. Yin, and H. Wang, First-principles investigation in the raman and infrared spectra of $\mathrm{sp}^{3}$ carbon allotropes, Carbon 78, 70 (2014).

[18] J.-C. Charlier, X. Gonze, and J.-P. Michenaud, Graphite Interplanar Bonding: Electronic Delocalization and van der Waals Interaction, Europhys. Lett. 28, 403 (1994).

[19] M. C. Schabel and J. L. Martins, Energetics of interplanar binding in graphite, Phys. Rev. B 46, 7185 (1992).

[20] M. Hasegawa, K. Nishidate, and H. Iyetomi, Energetics of interlayer binding in graphite: The semiempirical approach revisited, Phys. Rev. B 76, 115424 (2007).

[21] Y.-H. Kim, I.-H. Lee, S. Nagaraja, J.-P. Leburton, R. Q. Hood, and R. M. Martin, Two-dimensional limit of exchangecorrelation energy functional approximations, Phys. Rev. B 61, 5202 (2000).

[22] N. Mounet and N. Marzari, First-principles determination of the structural, vibrational and thermodynamic properties of diamond, graphite, and derivatives, Phys. Rev. B 71, 205214 (2005).

[23] T. Bučko, J. Hafner, S. Lebegue, and J. G. Angyán, Improved description of the structure of molecular and layered crystals: $a b$ initio DFT calculations with van der Waals corrections, J. Phys. Chem. A 114, 11814 (2010).

[24] T. Bučko, S. Lebègue, J. Hafner, and J. G. Ángyán, Tkatchenkoscheffler van der waals correction method with and without selfconsistent screening applied to solids, Phys. Rev. B 87, 064110 (2013)

[25] G. Graziano, J. Klimeš, F. Fernandez-Alonso, and A. Michaelides, Improved description of soft layered materials with van der Waals density functional theory, J. Phys.: Condens. Matter 24, 424216 (2012).
[26] T. Björkman, A. Gulans, A. V. Krasheninnikov, and R. M. Nieminen, Are we van der Waals ready? J. Phys.: Condens. Matter 24, 424218 (2012).

[27] T. Björkman, van der waals density functional for solids, Phys. Rev. B 86, 165109 (2012).

[28] C. R. C. Rêgo, L. N. Oliveira, P. Tereshchuk, and J. L. F. Da Silva, Comparative study of van der Waals corrections to the bulk properties of graphite, J. Phys.: Condens. Matter 27, 415502 (2015).

[29] W. Gao and A. Tkatchenko, Sliding Mechanisms in Multilayered Hexagonal Boron Nitride and Graphene: The Effects of Directionality, Thickness, and Sliding Constraints, Phys. Rev. Lett. 114, 096101 (2015).

[30] I. V. Lebedeva, A. V. Lebedev, A. M. Popov, and A. A. Knizhnik, Comparison of performance of van der Waals-corrected exchange-correlation functionals for interlayer interaction in graphene and hexagonal boron nitride, Comput. Mater. Sci. 128, 45 (2017).

[31] C. Lechner, B. Pannier, P. Baranek, N. C. Forero-Martinez, and H. Vach, First-principles study of the structural, electronic, dynamic, and mechanical properties of HOPG and diamond: Influence of exchange-correlation functionals and dispersion interactions, J. Phys. Chem. C 120, 5083 (2016).

[32] J. Klimeš, D. R. Bowler, and A. Michaelides, Van der Waals density functionals applied to solids, Phys. Rev. B 83, 195131 (2011).

[33] G.-X. Zhang, A. Tkatchenko, J. Paier, H. Appel, and M. Scheffler, Van Der Waals Interactions in Ionic and Semiconductor Solids, Phys. Rev. Lett. 107, 245501 (2011).

[34] W. A. Al-Saidi, V. K. Voora, and K. D. Jordan, An assessment of the vdW-TS method for extended systems, J. Chem. Theory Comput. 8, 1503 (2012).

[35] J. Park, B. D. Yu, and S. Hong, Van der Waals density functional theory study for bulk solids with BCC, FCC, and diamond structures, Curr. Appl. Phys. 15, 885 (2015).

[36] F. Tran, J. Stelzl, and P. Blaha, Rungs 1 to 4 of DFT Jacob's ladder: Extensive test on the lattice constant, bulk modulus, and cohesive energy of solids, J. Chem. Phys. 144, 204120 (2016).

[37] J. Tao, F. Zheng, J. Gebhardt, J. P. Perdew, and A. M. Rappe, Screened van der Waals correction to density functional theory for solids, Phys. Rev. Mater. 1, 020802 (2017).

[38] G.-X. Zhang, A. M. Reilly, A. Tkatchenko, and M. Scheffler, Performance of various density-functional approximations for cohesive properties of 64 bulk solids, New J. Phys. 20, 063020 (2018).

[39] C.-J. Yu, G.-C. Ri, U.-G. Jong, Y.-G. Choe, and S.-J. Cha, Refined phase coexistence line between graphite and diamond from density-functional theory and van der Waals correction, Physica B (Amsterdam) 434, 185 (2014).

[40] A. Tkatchenko, R. A. DiStasio, R. Car, and M. Scheffler, Accurate and Efficient Method for Many-Body Van Der Waals Interactions, Phys. Rev. Lett. 108, 236402 (2012).

[41] A. Ambrosetti, A. M. Reilly, R. A. DiStasio, Jr., and A. Tkatchenko, Long-range correlation energy calculated from coupled atomic response functions, J. Chem. Phys. 140, 18A508 (2014).

[42] S. Grimme, J. Antony, S. Ehrlich, and H. Krieg, A consistent and accurate $a b$ initio parametrization of density func- 
tional dispersion correction (DFT-D) for the 94 elements H-Pu, J. Chem. Phys. 132, 154104 (2010).

[43] S. Grimme, S. Ehrlich, and L. Goerigk, Effect of the damping function in dispersion corrected density functional theory, J. Comput. Chem. 32, 1456 (2011).

[44] A. Tkatchenko and M. Scheffler, Accurate Molecular Van Der Waals Interactions from Ground-State Electron Density and Free-Atom Reference Data, Phys. Rev. Lett. 102, 073005 (2009).

[45] J. Klimeš, D. R. Bowler, and A. Michaelides, Chemical accuracy for the van der Waals density functional, J. Phys.: Condens. Matter 22, 022201 (2010).

[46] I. Hamada, van der Waals density functional made accurate, Phys. Rev. B 89, 121103 (2014).

[47] O. A. Vydrov and T. Van Voorhis, Nonlocal Van Der Waals Density Functional Made Simple, Phys. Rev. Lett. 103, 063004 (2009).

[48] Y. Zhang and W. Yang, Comment on "Generalized Gradient Approximation Made Simple”, Phys. Rev. Lett. 80, 890 (1998).

[49] É. D. Murray, K. Lee, and D. C. Langreth, Investigation of exchange energy density functional accuracy for interacting molecules, J. Chem. Theory Comput. 5, 2754 (2009).

[50] A. D. Becke, On the large-gradient behavior of the density functional exchange energy, J. Chem. Phys. 85, 7184 (1986).

[51] K. Lee, É. D. Murray, L. Kong, B. I. Lundqvist, and D. C. Langreth, Higher-accuracy van der Waals density functional, Phys. Rev. B 82, 081101 (2010).

[52] P. E. Blöchl, Projector augmented-wave method, Phys. Rev. B 50, 17953 (1994).

[53] G. Kresse and D. Joubert, From ultrasoft pseudopotentials to the projector augmented-wave method, Phys. Rev. B 59, 1758 (1999).

[54] G. Kresse and J. Furthmüller, Efficient iterative schemes for ab initio total-energy calculations using a plane-wave basis set, Phys. Rev. B 54, 11169 (1996).

[55] G. Kresse and J. Hafner, Ab initio molecular-dynamics simulation of the liquid-metal-amorphous-semiconductor transition in germanium, Phys. Rev. B 49, 14251 (1994).

[56] G. Kresse and J. Hafner, Ab initio molecular dynamics for liquid metals, Phys. Rev. B 47, 558 (1993).

[57] G. Kresse and J. Furthmüller, Efficiency of ab-initio total energy calculations for metals and semiconductors using a planewave basis set, Comput. Mater. Sci. 6, 15 (1996).

[58] H. J. Monkhorst and J. D. Pack, Special points for Brillouinzone integrations, Phys. Rev. B 13, 5188 (1976).

[59] Y. Le Page and P. Saxe, Symmetry-general least-squares extraction of elastic data for strained materials from $a b$ initio calculations of stress, Phys. Rev. B 65, 104104 (2002).

[60] X. Wu, D. Vanderbilt, and D. R. Hamann, Systematic treatment of displacements, strains, and electric fields in densityfunctional perturbation theory, Phys. Rev. B 72, 035105 (2005).

[61] A. Togo, F. Oba, and I. Tanaka, First-principles calculations of the ferroelastic transition between rutile-type and $\mathrm{CaCl}_{2}$-type $\mathrm{SiO}_{2}$ at high pressures, Phys. Rev. B 78, 134106 (2008).

[62] T. Bučko, S. Lebègue, J. G. Ángyán, and J. Hafner, Extending the applicability of the Tkatchenko-Scheffler dispersion correction via iterative Hirshfeld partitioning, J. Chem. Phys. 141, 034114 (2014).

[63] Y. Baskin and L. Meyer, Lattice Constants of Graphite at Low Temperatures, Phys. Rev. 100, 544 (1955).

[64] Y. X. Zhao and I. L. Spain, X-ray diffraction data for graphite to 20 GPa, Phys. Rev. B 40, 993 (1989).

[65] G. I. Csonka, J. P. Perdew, A. Ruzsinszky, P. H. T. Philipsen, S. Lebègue, J. Paier, O. A. Vydrov, and J. G. Ángyán, Assessing the performance of recent density functionals for bulk solids, Phys. Rev. B 79, 155107 (2009).

[66] P. Gillet, G. Fiquet, I. Daniel, B. Reynard, and M. Hanfland, Equations of state of $12 \mathrm{c}$ and $13 \mathrm{c}$ diamond, Phys. Rev. B 60, 14660 (1999).

[67] A. Bosak, M. Krisch, M. Mohr, J. Maultzsch, and C. Thomsen, Elasticity of single-crystalline graphite: Inelastic X-ray scattering study, Phys. Rev. B 75, 153408 (2007).

[68] H. J. McSkimin and P. Andreatch, Jr., Elastic moduli of diamond as a function of pressure and temperature, J. Appl. Phys. 43, 2944 (1972).

[69] G. Savini, Y. J. Dappe, S. Öberg, J.-C. Charlier, M. I. Katsnelson, and A. Fasolino, Bending modes, elastic constants and mechanical stability of graphitic systems, Carbon 49,62 (2011).

[70] J. B. Nelson and D. P. Riley, The thermal expansion of graphite from $15^{\circ}$ c. to $800^{\circ}$ c.: part I. Experimental, Proc. Phys. Soc. 57, 477 (1945).

[71] Q. Y. Lin, T. Q. Li, Z. J. Liu, Y. Song, L. L. He, Z. J. Hu, Q. G. Guo, and H. Q. Ye, High-resolution TEM observations of isolated rhombohedral crystallites in graphite blocks, Carbon 50, 2369 (2012).

[72] H. A. Wilhelm, B. Croset, and G. Medjahdi, Proportion and dispersion of rhombohedral sequences in the hexagonal structure of graphite powders, Carbon 45, 2356 (2007).

[73] R. Z Khaliullin, H. Eshet, T. D. Kühne, J. Behler, and M. Parrinello, Nucleation mechanism for the direct graphite-todiamond phase transition, Nat. Mater. 10, 693 (2011).

[74] S. Lebègue, J. Harl, T. Gould, J. G. Ángyán, G. Kresse, and J. F. Dobson, Cohesive Properties and Asymptotics of the Dispersion Interaction in Graphite by the Random Phase Approximation, Phys. Rev. Lett. 105, 196401 (2010).

[75] C. S. G. Cousins, Elasticity of carbon allotropes. IV. Rhombohedral graphite: Elasticity, zone-center optic modes, and phase transformation using transferred Keating parameters, Phys. Rev. B 67, 024110 (2003).

[76] R. Zacharia, H. Ulbricht, and T. Hertel, Interlayer cohesive energy of graphite from thermal desorption of polyaromatic hydrocarbons, Phys. Rev. B 69, 155406 (2004).

[77] Z. Liu, J. Z. Liu, Y. Cheng, Z. Li, L. Wang, and Q. Zheng, Interlayer binding energy of graphite: A mesoscopic determination from deformation, Phys. Rev. B 85, 205418 (2012). 